University of Nebraska - Lincoln

DigitalCommons@University of Nebraska - Lincoln

2003

\title{
Sodium tanshinone IIA sulfonate mediates electron transfer reaction in rat heart mitochondria
}

\author{
Guangyin Zhou \\ Institute of Biophysics \\ Wen Jiang \\ Institute of Biophysics \\ Yan Zhao \\ Institute of Biophysics \\ Guangén Ma \\ The National Institute of Pharmaceutical Research and Development \\ Wenjuan Xin \\ Institute of Biophysics \\ See next page for additional authors
}

Follow this and additional works at: https://digitalcommons.unl.edu/publichealthresources

Part of the Public Health Commons

Zhou, Guangyin; Jiang, Wen; Zhao, Yan; Ma, Guangén; Xin, Wenjuan; Yin, Junjie; and Zhao, Baolu, "Sodium tanshinone IIA sulfonate mediates electron transfer reaction in rat heart mitochondria" (2003). Public Health Resources. 107.

https://digitalcommons.unl.edu/publichealthresources/107

This Article is brought to you for free and open access by the Public Health Resources at DigitalCommons@University of Nebraska - Lincoln. It has been accepted for inclusion in Public Health Resources by an authorized administrator of DigitalCommons@University of Nebraska - Lincoln. 


\section{Authors}

Guangyin Zhou, Wen Jiang, Yan Zhao, Guangén Ma, Wenjuan Xin, Junjie Yin, and Baolu Zhao 


\title{
Sodium tanshinone IIA sulfonate mediates electron transfer reaction in rat heart mitochondria
}

\author{
Guangyin Zhou ${ }^{\mathrm{a}}$, Wen Jiang ${ }^{\mathrm{a}}$, Yan Zhao ${ }^{\mathrm{a}}$, Guangén Ma ${ }^{\mathrm{b}}$, Wenjuan Xin ${ }^{\mathrm{a}}$, \\ Junjie Yin ${ }^{c}$, Baolu Zhao ${ }^{\text {a** }}$ \\ ${ }^{a}$ Laboratory of Visual Information Processing, Department of Molecular and Cell Biophysics, Institute of Biophysics, \\ Academia Sinica, Beijing 100101, PR China \\ ${ }^{\mathrm{b}}$ The National Institute of Pharmaceutical Research and Development, Beijing 102206, PR China \\ ${ }^{\mathrm{c}}$ Instrumentation and Biophysics Branch, CFSAN, FDA, 5100 Paint Branch Parkway, College Park, MD 20740, USA
}

Received 22 May 2002; accepted 6 September 2002

\begin{abstract}
In this paper, an electron transfer reaction mediated by sodium tanshinone IIA sulfonate (STS) was studied in rat heart mitochondria. It was found that STS could stimulate mitochondrial NADH oxidation dose-dependently and partly restore NADH oxidation in the presence of respiratory inhibitor (rotenone or antimycin A or $\mathrm{KCN}$ ). It was likely that STS could accept electrons from complex I similar to ferricyanide and could be converted to its semiquinone form that could then reduce oxygen molecule. The data also showed that cytochrome $c$ (Cyt c) could be reduced by STS in the presence of KCN, or STS could transfer the electron to oxygen directly. Free radicals were involved in the process. The results suggest that STS may protect ischemia-reperfusion injury through an electron transfer reaction in mitochondria against forming reactive oxygen radicals.
\end{abstract}

(C) 2002 Elsevier Science Inc. All rights reserved.

Keywords: Sodium tanshinone IIA sulfonate (STS); Mitochondria; Respiratory chain; Free radicals; Antioxidant; Electron spin resonance (ESR)

\section{Introduction}

Salvia miltiorrhiza is a Chinese traditional medicine and has long been used for treating heart disease in China. Sodium tanshinone IIA sulfonate, a water soluble derivative of tanshinone IIA, is the main effective component of $S$. miltiorrhiza. The molecular structure of STS is depicted in Fig. 1. Clinical evidence has shown that STS increases coronary blood flow, alleviates cardiac metabolic disorders and protects heart against ischemia-reperfusion injury [1,2]. Several mechanisms have been proposed for the protective effects of STS on ischemia-reperfusion injury: (1) STS acts as an antioxidant that can scavenge free radicals [3-5]; (2) STS is an inhibitor of heart calcium channel, which can inhibit $\mathrm{Ca}^{2+}$ overload [6]; (3) STS inhibits the phagocyte

\footnotetext{
* Corresponding author. Tel.: +86-10-64888569; fax: +86-10-64871293.

E-mail address: zhaobl@sun5.ibp.ac.cn (B. Zhao).

Abbreviations: STS, sodium tanshinone IIA sulfonate; ESR, electron spin resonance; NADH, $\beta$-nicotinamide adenine dinucleotide; SOD, superoxide dismutase; DMPO, 5,5-dimethyl-1-pyrroline- $N$-oxide; AA, antimycin A; Cyt c, cytochrome $c$; ROS, reactive oxygen species.
}

adhesion, phagocytosis and acid-phosphatase release from neutrophils [7]. The increase of NADH level caused by anaerobic glycolysis is reported to stimulate $\mathrm{O}_{2}{ }^{--}$production during reperfusion, causing dysfunction of cytochrome oxidase and an increase of electron leakage [8]. Many clinically important chemotherapeutic drugs have a quinone nucleus in their molecules. Redox cycling of quinones catalyzed by one-electron transfer enzymes leads to the production of reactive oxygen species (ROS) $[9,10]$. For example, adriamycin can accept an electron from mitochondrial complex I and form a semiquinone free radical. This semiquinone radical then reacts with oxygen and forms $\mathrm{O}_{2}{ }^{\bullet-}$, causing cardiotoxicity [11]. STS is a derivative of phenanthrenequinone, and it may act as an electron acceptor at mitochondrial complex I, NADH dehydrogenase. However, the effects of STS on mitochondrial respiratory chain in the presence of NADH have never been studied.

Oxidative phosphorylation takes place in mitochondria at which, substrates are oxidized and the electrochemical gradients generated are used to synthesize ATP. In heart, mitochondria play a critical role since the work of heart requires a great amount of energy. At the same time, 


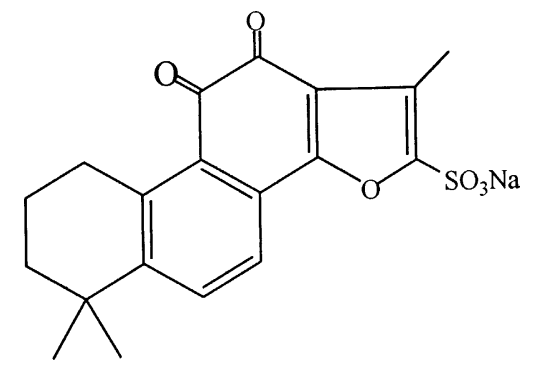

Fig. 1. Molecular structure of sodium tanshinone IIA sulfonate.

mitochondria are the major place for free radical generation. The production of ROS and the related damage are the main cause of injury in the process of ischemia-reperfusion $[12,13]$. In this study, the effects of STS on the mitochondrial respiratory chain were investigated by using NADH as a substrate. Our data reveal that STS stimulates NADH oxidation in mitochondria accepting electrons at complex I by acting as a redox cycler, suggesting that a therapeutical potential of STS may be utilized to protect ischemiareperfusion injury.

\section{Materials and methods}

\subsection{Reagents}

Sodium tanshinone IIA sulfonate was isolated and purified in The National Institutes of Pharmaceutical Research and Development. 5,5-Dimethyl-1-pyrroline- $\mathrm{N}$-oxide (DMPO), rotenone, antimycin A, NADH ( $\beta$-nicotinamide adenine dinucleotide, reduced form), luminol (5-amino2,3-dihydro-1,4-phthalazinedione), superoxide dismutase (SOD, from bovine blood) and catalase (from horse liver) were obtained from Sigma. DMPO was purified by active charcoal. All other reagents made in China were of AR level.

\subsection{Mitochondrial preparation}

Normal mitochondria from male Wistar rats (200$250 \mathrm{~g}$ ) were isolated as described previously [14] with slight modifications. Isolated hearts were homogenized in medium containing $0.3 \mathrm{M}$ sucrose, $1 \mathrm{mM}$ EDTA and $10 \mathrm{mM}$ Tris- $\mathrm{HCl}$ (pH 7.4). The homogenates were centrifuged at $600 \mathrm{~g}$ for $10 \mathrm{~min}$. The supernatants were collected and centrifuged at $10,000 \mathrm{~g}$ for $10 \mathrm{~min}$. The pellets containing fresh mitochondria were washed twice and resuspended in the isolating solution and stored at $-20^{\circ}$. The mitochondria were thawed at $0^{\circ}$ before using. The preparations of mitochondria used as mitochondria according to the references [15-17] are permeable to NADH and some small molecule substrates. The morphologies of the mitochondrial preparations of both fresh and the "freezethaw" mitochondria were checked by an electron microscope and no difference was found. This process destroyed the respiratory control. The protein concentration was determined by Biuret method using BSA as standard [18].

\subsection{Effect of STS on NADH oxidation}

Effects of STS on NADH oxidation were determined by measuring the decrease of absorbance at $340 \mathrm{~nm}(\varepsilon=6.2$ / $\mathrm{mM} \mathrm{cm}$ ) using a Beckman DU-640 UV-Vis spectrophotometer at $37^{\circ}$. The system contained $0.3 \mathrm{~mol} / \mathrm{L}$ sucrose, $1 \mathrm{mM}$ EDTA and $10 \mathrm{mM}$ Tris-HCl, $0.1 \mathrm{mg}$ protein $/ \mathrm{mL}$ mitochondria and various concentrations of STS, $\mathrm{pH}$ 7.4. The reaction was performed by adding $0.2 \mathrm{mM}$ NADH to the mixture. The inhibition experiments were performed in the presence of $2 \mu \mathrm{M}$ rotenone, $2 \mu \mathrm{M}$ antimycin $\mathrm{A}$ or $1 \mu \mathrm{M}$ $\mathrm{KCN}$.

\subsection{Effect of STS on reduction of ferricyanide by mitochondrial complex I}

The reaction mixture contained $0.3 \mathrm{~mol} / \mathrm{L}$ sucrose, $1 \mathrm{mM}$ EDTA, $10 \mathrm{mM}$ Tris- $\mathrm{HCl}, 0.2 \mathrm{mM}$ NADH, $2 \mu \mathrm{M}$ rotenone, $1 \mathrm{mM} \mathrm{K}_{3}\left[\mathrm{Fe}(\mathrm{CN})_{6}\right], \quad 0.1 \mathrm{mg}$ protein $/ \mathrm{mL}$ mitochondria and various concentrations of STS, $\mathrm{pH}$ 7.4. Ferricyanide reduction was determined by measuring the change of absorbance at $420 \mathrm{~nm}$ after the addition of NADH.

\subsection{Effect of STS on chemiluminescence released by mitochondria}

Luminol-dependent chemiluminescence was determined using a BPCL superweak chemiluminescemeter. The reaction mixture included: $0.3 \mathrm{mg} / \mathrm{mL}$ mitochondria, $0.2 \mathrm{mM}$ STS, 0.05 or $0.2 \mathrm{mM} \mathrm{NADH}, 0.2 \mathrm{mM}$ luminol and/or $500 \mathrm{U} / \mathrm{mL} \mathrm{SOD,} \mathrm{and/or} 500 \mathrm{U} / \mathrm{mL}$ catalase, and/or $50 \mathrm{mM}$ ethanol.

\subsection{Analysis of hydroxyl radical by ESR}

The analytical solution contained $1 \mathrm{mg}$ protein $/ \mathrm{mL}$ mitochondria, $1 \mathrm{mM}$ NADH, $0.2 \mathrm{mM}$ STS, $10 \mu \mathrm{M}$ rotenone or $10 \mu \mathrm{M}$ antimycin $\mathrm{A}$ or $1 \mathrm{mM} \mathrm{KCN}, 80 \mathrm{mM}$ DMPO, $50 \mathrm{mM}$ ethanol, $50 \mathrm{mM}$ potassium phosphate buffer, $\mathrm{pH}$ 7.4. The sample was mixed and transferred into a quartz capillary placed in the cavity of Varian E109C ESR spectrometer. The machine setting was: $\mathrm{X}$ band, microwave power $10 \mathrm{~mW}$, sweep width $200 \mathrm{G}$, modulation frequency $100 \mathrm{kHz}$, modulation amplitude $2 \mathrm{G}$, time constant $0.125 \mathrm{~s}$, scanning rate $50 \mathrm{G} / \mathrm{min}$.

\subsection{Measurement of STS radical by ESR}

STS was reduced by $1 \mathrm{mM} \mathrm{NaBH}_{4}$ and the $\mathrm{pH}$ of the reaction mixture was adjusted by adding $0.1 \mathrm{mM} \mathrm{NaOH}$. The mixture was transferred into a capillary for measuring ESR spectrum. The ESR conditions were: microwave power $0.2 \mathrm{~mW}, 100 \mathrm{kHz}$ modulation with amplitude 
$0.02 \mathrm{G}$, sweep width $20 \mathrm{G}$ with speed of $2.5 \mathrm{G} / \mathrm{min}$. Other conditions were the same as mentioned earlier.

\subsection{Measurement of reaction of STS with hydroxyl radical by pulse radiolysis}

The pulse radiolysis experiment was conducted using a line accelerator providing a $5 \mathrm{MeV}$ electron pulse with a duration of $2 \mu \mathrm{s}$ at room temperature. The average dose/ pulse was $5 \mathrm{~Gy}$ and the reaction was monitored by a $500 \mathrm{~W}$ xenon lamp. Hydroxyl radicals were generated from the pulsed $\mathrm{N}_{2} \mathrm{O}$ saturated $10 \mathrm{mM} \mathrm{KSCN}$ aqueous solution.

\subsection{Statistics}

The data were expressed as mean \pm SEM and analyzed by ANOVA. Comparison between the treatment groups was made by analyzing data with Newman-Keuls test. $P$-values less than 0.05 were considered statistically significant.

\section{Results}

\subsection{Effects of STS on the oxidation of NADH by mitochondrial transport chain}

The oxidation of NADH was monitored by the decrease of optical density (O.D.) value at $340 \mathrm{~nm}$. The O.D. value decreased rapidly when fresh isolated heart mitochondria were added to the reaction mixture, indicating that NADH was oxidized by heart mitochondria via the respiratory chain (details not shown).

Fig. 2 shows the effects of STS on NADH oxidation in mitochondria treated with various respiratory chain inhibitors. When mitochondria were treated with rotenone, antimycin $\mathrm{A}$ or $\mathrm{KCN}$, the rate of the NADH oxidation was decreased nearly to zero. Addition of STS partially restored the oxidation of $\mathrm{NADH}$ to approximately the same levels as that in the absence of all three above inhibitors. STS did not directly react with NADH in a control experiment (details not shown).

The relation between the amount of the oxidized NADH caused by STS and the amount of added STS was investigated in rotenone-inhibited mitochondria. NADH was added again after previous NADH was oxidized. This process was repeated several times; all added NADH had been oxidized at a stable rate whereas the amount of oxidized NADH was 10 times more than that of original NADH after STS treatment.

The storage of fresh mitochondria at $-20^{\circ}$ did not cause a dysfunction of the mitochondrial respiratory chain as similar results were obtained using fresh isolated mitochondria.

STS stimulated mitochondrial NADH oxidation was dose-dependent and the oxidation rate reached to a maximum at about $0.3 \mathrm{mM}$, as shown in Fig. 3. This effect was also observed in the presence of respiratory inhibitors. When the respiratory chain was blocked by rotenone, the rate of NADH oxidation decreased nearly to zero. Addition of STS to the system restored NADH oxidation in a dosedependent manner, and a maximum rate was observed at concentration of $0.3 \mathrm{mM}$. Similar result was obtained in the presence of antimycin A or KCN. Rotenone blocks electron flow from complex I to ubiquinone, antimycin A blocks complex III, and KCN blocks electron flow from Cyt $c$ to Cyt $\mathrm{a}_{3}$. STS could partly reverse the effects of these three inhibitors, indicating that a new bifurcated electron pathway might be formed by STS at the site of complex I, probably through flavoprotein which is located before the sensitive site of rotenone.

\subsection{Inhibitory effect of STS on $\mathrm{K}_{3}\left[\mathrm{Fe}(\mathrm{CN})_{6}\right]$ reduction in mitochondria}

$\mathrm{K}_{3}\left[\mathrm{Fe}(\mathrm{CN})_{6}\right]$ is an artificial electron acceptor which can accept electrons at mitochondrial complex I, causing a

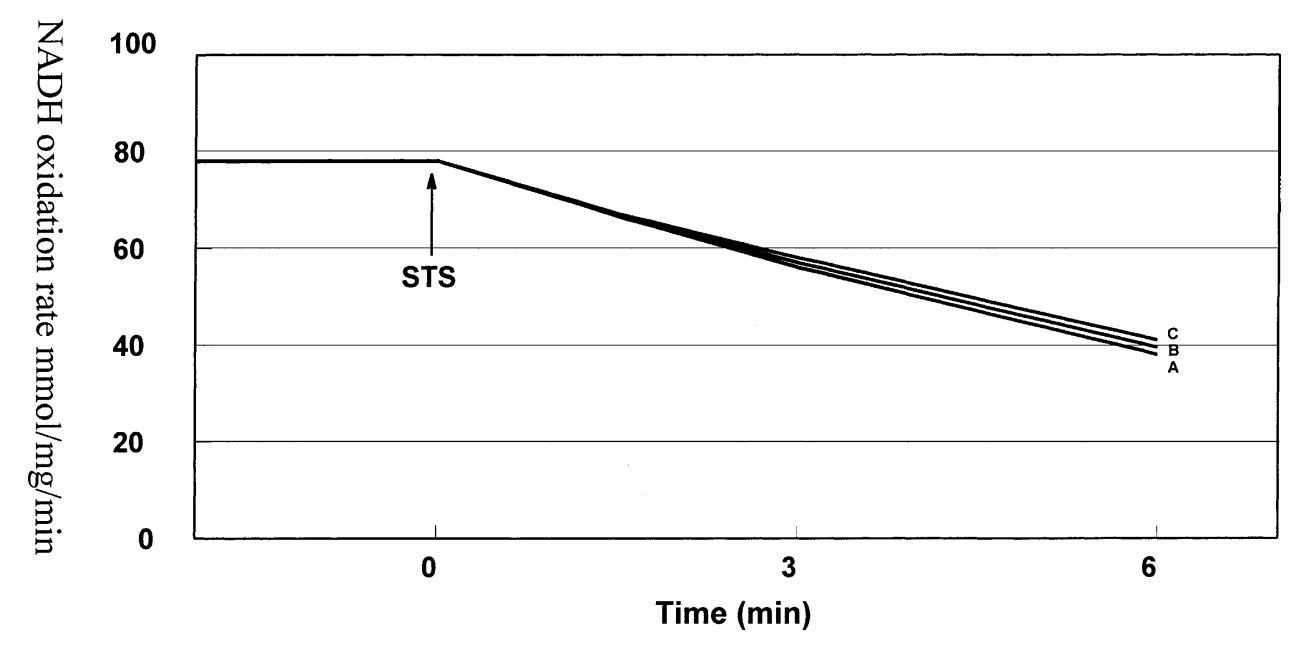

Fig. 2. Recovering effects of STS on NADH oxidation in inhibited mitochondria. The solution contained $0.3 \mathrm{mg}$ protein/mL fresh mitochondria, $0.5 \mathrm{mM}$ STS, $1.25 \mathrm{mM} \mathrm{NADH}$ and in the presence of (A) $2 \mathrm{mM}$ rotenone; (B) $2 \mathrm{mM}$ antimycin A; (C) $1 \mathrm{mM} \mathrm{KCN}$. 


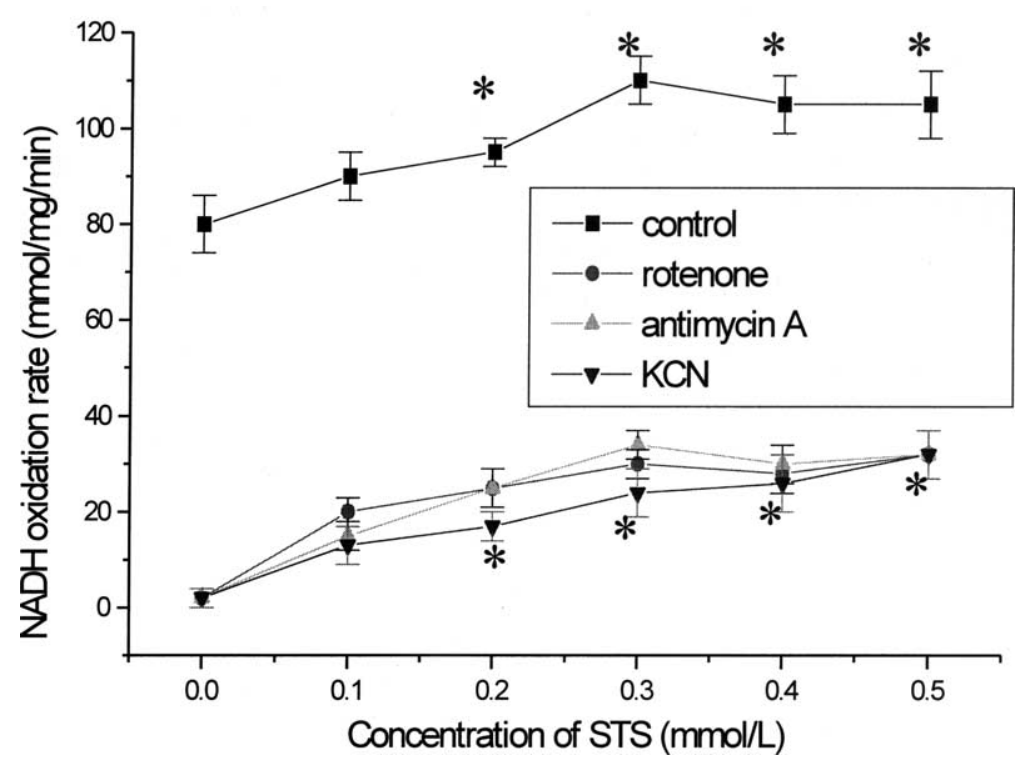

Fig. 3. Effect of STS on NADH consumption in the presence or absence of inhibitors. NADH consumption was measured at $340 \mathrm{~nm}$. Final concentrations were: $0.3 \mathrm{mg}$ protein/mL mitochondria, $0.3 \mathrm{M}$ sucrose, $0.1 \mathrm{mM}$ EDTA, $10 \mathrm{mM}$ Tris- $\mathrm{HCl}, 0.2 \mathrm{mM}$ NADH, without inhibitors ( $\mathbf{\square})$, in the presence of rotenone $2 \mu \mathrm{M}(\bullet)$, antimycin A $2 \mu \mathrm{M}(\mathbf{A})$, or $\mathrm{KCN} 1 \mathrm{mM}(\boldsymbol{\nabla})$. Temperature was $37^{\circ}, \mathrm{pH}$ 7.4. Data are mean $\pm \mathrm{SD}$ of three experiments. $(*) P<0.05$ compared with that at $0 \mathrm{~min}$.

decrease absorbance at $420 \mathrm{~nm}$ [19]. Here we have shown that STS inhibited this reduction dose-dependently (Fig. 4). STS did not react with $\mathrm{K}_{3}\left[\mathrm{Fe}(\mathrm{CN})_{6}\right]$ directly, as demonstrated in a control experiment. Thus, STS may accept electrons from complex I similar to ferricyanide and its electron accepting ability is stronger than that of $\mathrm{K}_{3}\left[\mathrm{Fe}(\mathrm{CN})_{6}\right]$.

\subsection{STS induced mitochondria to release chemiluminescence}

When NADH was used as a substrate, an increase of chemiluminescence from mitochondria was observed in the presence of STS. Addition of NADH again caused a second enhancement of chemiluminescence (Fig. 5).

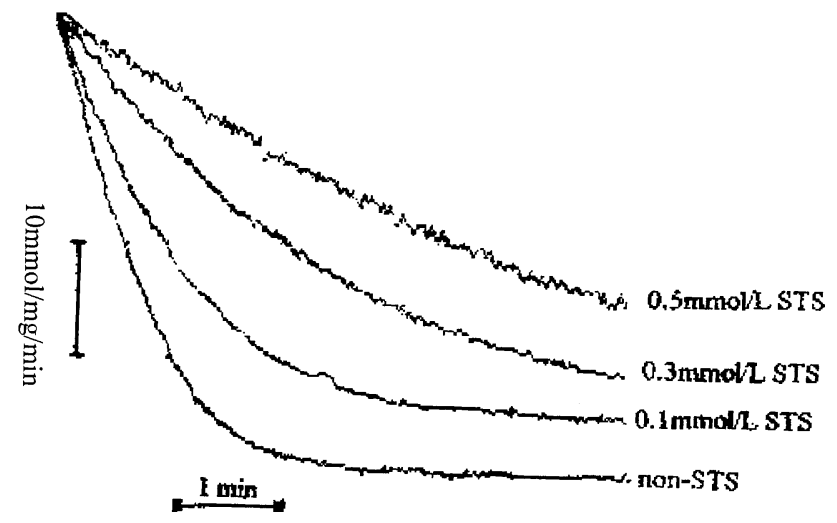

Fig. 4. Effect of STS on ferricycanide reduction by heart mitochondrial complex I. Ferricyanide reduction was measured at $420 \mathrm{~nm}$ after the addition of NADH to the reaction system. Final concentration: $0.3 \mathrm{mg}$ protein $/ \mathrm{mL}$ mitochondria, $0.2 \mathrm{mM}$ NADH, $1 \mathrm{mM}$ ferricyanide, $2 \mu \mathrm{M}$ rotenone. The lines were drawn from the same point of origin for comparison.
When rotenone or antimycin A was used as respiratory inhibitor, chemiluminescence was increased but the time was delayed. When $\mathrm{KCN}$ was used, a similar result was obtained except that the chemiluminescence intensity was increased about 12 times of that in the presence of rotenone or antimycin A. These results clearly showed that ROS were formed during STS-mediated electron transfers, especially in the presence of $\mathrm{KCN}$. The reaction of the reduced intermediate with Cyt $\mathrm{c}$ was also studied by excess ferricytochrome $c$ in the incubation and monitoring its reduction in the presence of $\mathrm{KCN}$. It was found that ferricytochrome $c$ could be reduced and if SOD was added to the system, the reduction was decreased.

To determine which free radicals were involved in this process, several scavengers were added to the system. Fig. 6 is a typical chemiluminescence curve of STS-mediated pathway in the presence of rotenone. SOD eliminated the chemiluminescence completely; catalase or ethanol only partially eliminated the chemiluminescence. These data suggest that $\mathrm{O}_{2}{ }^{\bullet-}, \mathrm{H}_{2} \mathrm{O}_{2}$ and $\mathrm{OH}^{\bullet}$ contributed to the chemiluminescence, but only $\mathrm{O}_{2}{ }^{\bullet-}$ was the primary free radical formed in this process.

\subsection{Effect of STS on hydroxyl radical generated from inhibitory respiratory chain}

When rotenone or antimycin A was used as inhibitor in the presence of STS, no free radical was trapped by DMPO. When KCN was used, hydroxyl free radical signal was observed as indicated by four peaks with intensities $1: 2: 2: 1\left(a_{\mathrm{N}}=a_{\mathrm{H}}=14.9 \mathrm{G}\right) \quad$ (Fig. 7B). Excess ethanol quenched almost all the DMPO-OH spectrum in parallel with the appearance of the hydroxyethyl adducts as 


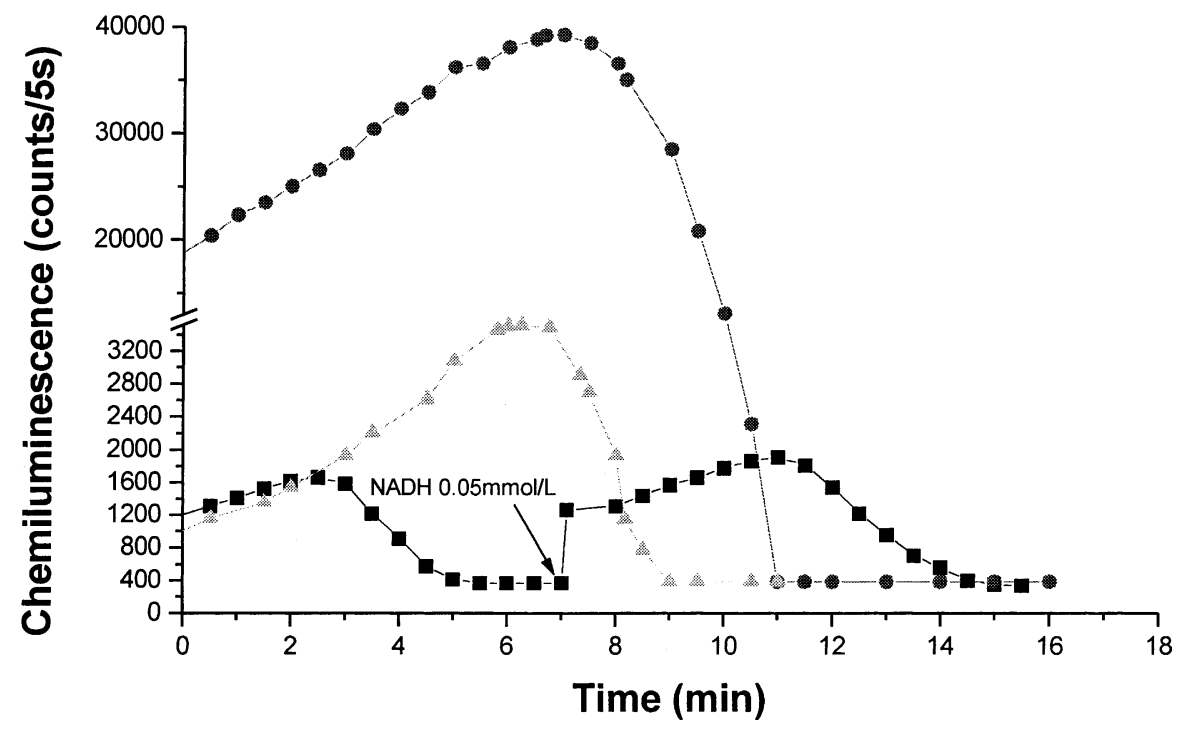

Fig. 5. STS-induced mitochondrial chemiluminescence by addition of NADH and effects of respiratory inhibitors. The measuring system contained: $0.3 \mathrm{mg}$ protein/mL mitochondria, $0.2 \mathrm{mM}$ STS, $0.2 \mathrm{mM}$ luminol, $0.3 \mathrm{M}$ sucrose, $10 \mathrm{mM} \mathrm{HEPES}$, with $0.05 \mathrm{mM}$ NADH as substrate (pH 7.4) in the presence of $1 \mathrm{mM}$ $\mathrm{KCN}(\mathbf{O})$; in the presence of $2 \mu \mathrm{M}$ rotenone $(\boldsymbol{\square})$; or in the presence of antimycin A (A). Temperature, $37^{\circ}$.

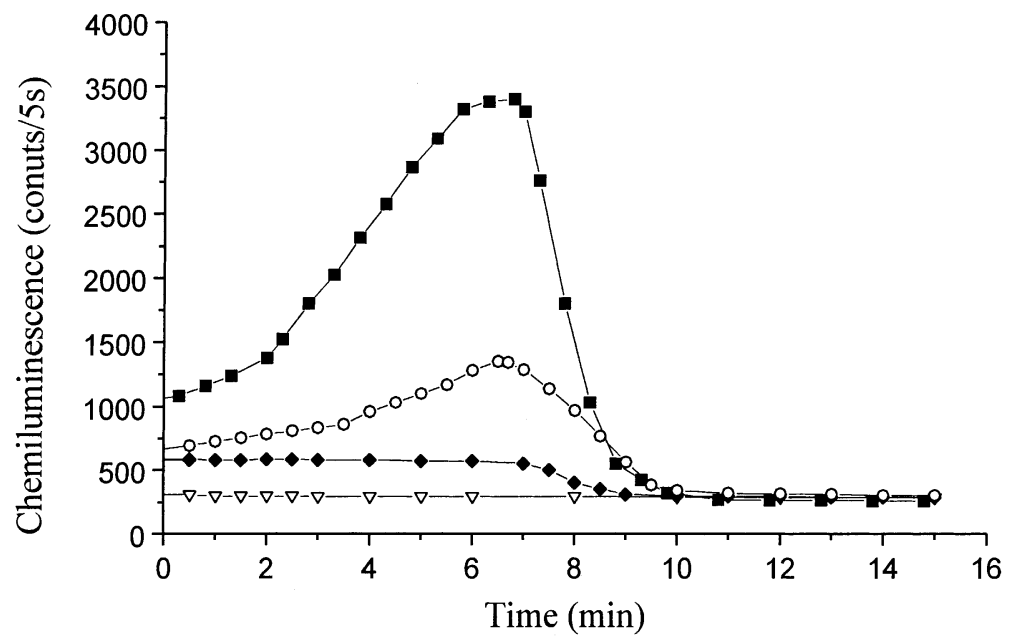

Fig. 6. Effects of free radical scavengers on the STS-induced chemiluminescence in mitochondria. The system contained: $2 \mu \mathrm{M}$ rotenone, $0.3 \mathrm{mg} / \mathrm{mL}$

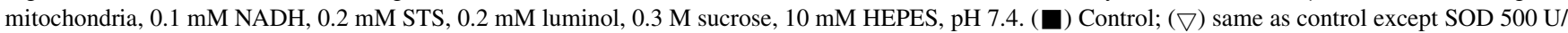
$\mathrm{mL}$ was added; $(\diamond)$ same as control except catalase $500 \mathrm{U} / \mathrm{mL}$ was added; $(\bigcirc)$ same as control except ethanol $50 \mathrm{mM}$ was added. Temperature, $37^{\circ}$.

indicated by six peaks with intensities $1: 1: 1: 1: 1: 1\left(a_{\mathrm{N}}=\right.$ $\left.15.6 \mathrm{G}, a_{\mathrm{H}}=22.5 \mathrm{G}\right)($ Fig. 7A) [20]. The adduct of DMPO with $\mathrm{O}_{2}{ }^{--}$was not obtained, probably because it decomposed to DMPO-OH. However, the adduct of DMPO-OH was generated, at least in part, via $\mathrm{O}_{2}{ }^{\bullet-}$, because addition of SOD could quench all the signals. These data suggested that free radicals were involved in STS-mediated pathway, especially when $\mathrm{KCN}$ was used as an inhibitor.

\subsection{STS free radical}

The STS free radical was examined by ESR spin trapping technique in above system but no ESR signal was found. If STS was first reduced by $\mathrm{NaBH}_{4}(1 \mathrm{mmol} / \mathrm{L})$ and then reoxidized by oxygen in alkaline condition, an absorption peak at $300 \mathrm{~nm}$ and an ESR signal of free radical were found. Analysis of the ESR spectrum of the reoxidized sample revealed that it was the semiquinone free radical of STS (Fig. 8). Using pulse radiolysis technique, it was found that there was a transient absorption peak at $300 \mathrm{~nm}$ in the system of reaction of STS with hydroxyl radical, and the reaction constant between hydroxyl radical and STS was calculated to be approximately $1.8 \times 10^{9} \mathrm{dm}^{3} / \mathrm{mol} \mathrm{s}$.

\section{Discussion}

This is the first report that STS can act as an electron carrier and mediate an electron transfer. A possible mechanism for STS action is shown in Fig. 9. STS accepts electrons from mitochondrial complex I, probably through flavoprotein, which is located before the sensitive site of 


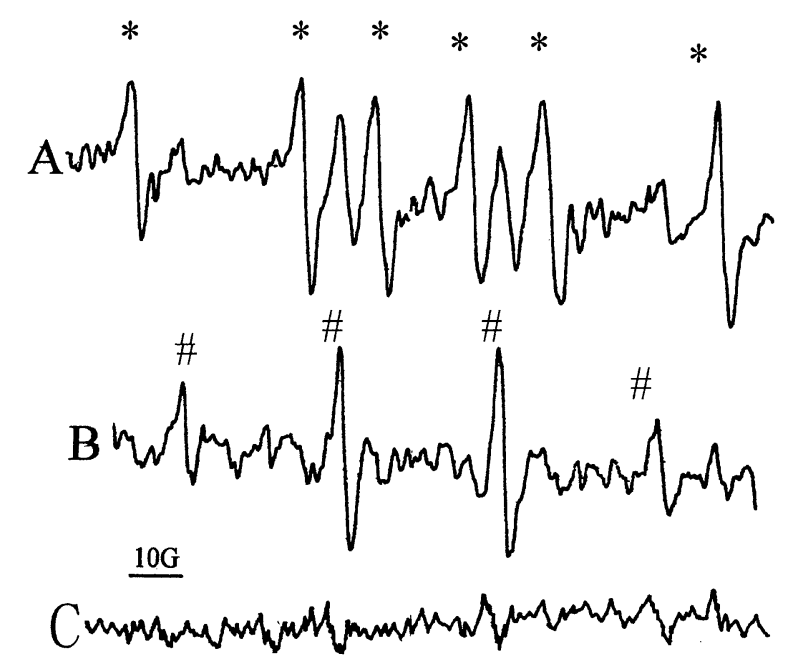

Fig. 7. The ESR spectrum of adduct of DMPO and free radical produced by rat heart mitochondria. (A) The adducts DMPO- $\mathrm{CH}(\mathrm{OH}) \mathrm{CH}_{3}\left(a_{\mathrm{N}}=\right.$ $15.8 \mathrm{G}, a_{\mathrm{H}}=22.5 \mathrm{G}$ ) (indicated by $*$ ) generated from the respiratory chain inhibited by $\mathrm{KCN}$ in the presence of NADH and STS with excess ethanol; (B) the adduct DMPO-OH $\left(a_{\mathrm{N}}=a_{\mathrm{H}}=14.9 \mathrm{G}\right)$ (indicated by \#) generated from respiratory chain inhibited by $\mathrm{KCN}$ in the presence of NADH and STS; (C) control spectrum from the mixture of mitochondria with DMPO without inhibition of $\mathrm{KCN}$. The machine setting were: $\mathrm{X}$ band, microwave $10 \mathrm{~mW}$ sweep width $200 \mathrm{G}$, modulation frequency $100 \mathrm{kHz}$, modulation amplitude $2 \mathrm{G}$, time constant $0.128 \mathrm{~s}$, scanning rate $50 \mathrm{G} / \mathrm{min}$ at room temperature.

rotenone, and facilitates an electron transfer. NADH is oxidized by STS-mediated pathway in the presence of rotenone, antimycin or KCN. In this pathway, electrons may be transferred directly to oxygen in the presence of $\mathrm{KCN}$, because its sensitive site is complex IV. However, it is not clear if the electron reenters respiratory chain again, at complex III Cyt c or complex IV in the presence of antimycin A or rotenone, and in the absence of $\mathrm{KCN}$. To address these questions, ROS formation during this process was examined by using chemiluminescence. The chemiluminescence is a sensitive method often used to study the formation of ROS in biological systems. It was found that when mitochondria were blocked by rotenone or antimycin $\mathrm{A}$, the chemiluminscence intensity was increased along with NADH oxidation. A similar result was obtained when $\mathrm{KCN}$ was used as inhibitor except that the intensity of chemiluminescence was about 12 times higher than that induced by rotenone or antimycin A. It was also examined if the reduced intermediate could react with Cyt $\mathrm{c}$ by testing the reduction of excess ferricytochrome $c$ in the

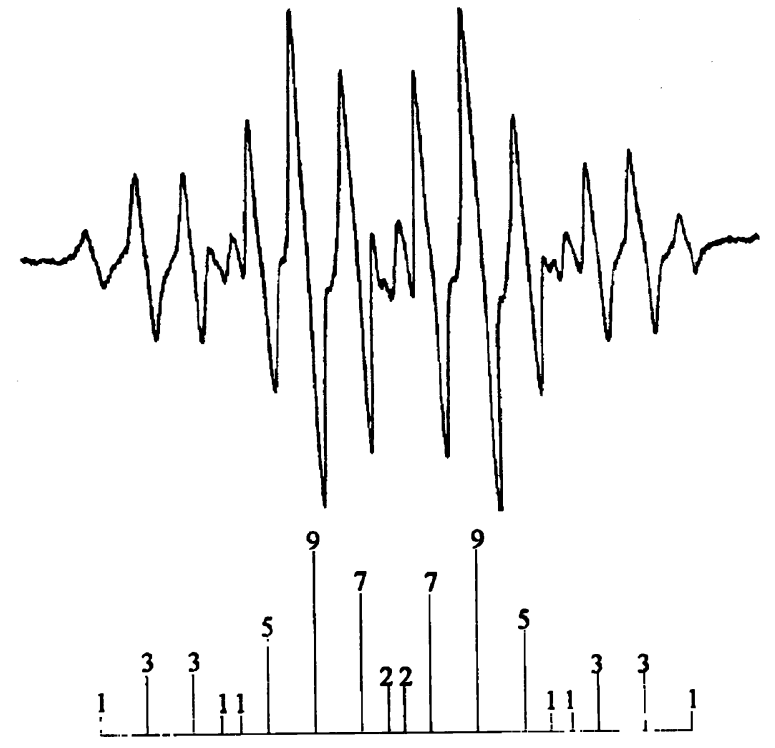

Fig. 8. ESR spectrum of semiquinone free radical of STS. The mixture contained $1 \mathrm{mM}$ STS, $1 \mathrm{mM} \mathrm{NaBH} 4$ and $0.1 \mathrm{mM} \mathrm{NaOH}$. The ESR conditions were: microwave power $0.2 \mathrm{~mW}, 100 \mathrm{kHz}$ modulation with amplitude $0.02 \mathrm{G}$, sweep width $20 \mathrm{G}$ with speed of $2.5 \mathrm{G} / \mathrm{min}$.

incubation system in the presence of KCN. If SOD was added to the system, the reduction was decreased because $\mathrm{O}_{2}{ }^{\bullet-}$ was scavenged. This difference could be explained by the alternative electron transfer in the STS-mediated pathway. The inhibiting site for $\mathrm{KCN}$ is downstream of $\mathrm{Cyt}$ c; therefore STS provides electrons to oxygen by a direct pathway. $\mathrm{O}_{2}{ }^{\bullet-}, \mathrm{H}_{2} \mathrm{O}_{2}$, and hydroxyl radical might be formed during this process, which is the cause for the very strong chemiluminescence. However, when rotenone or antimycin A was used as inhibitor in the absence of $\mathrm{KCN}$, electrons passing through an STS-mediated pathway might enter the respiratory chain again insofar as the sensitive sites of these two inhibitors are upstream of Cyt c; this process, less ROS were generated and, therefore, a low chemiluminescence was observed.

NADH dehydrogenase residing in mitochondrial complex I can divert electrons from NADH to an exogenous quinone and form a semiquinone free radical [8-13]. This semiquinone can be oxidized by oxygen. In this study, we demonstrated that free radicals were involved in this process. SOD inhibited the chemiluminescene completely, while catalase and ethanol only partly inhibited the chemiluminescence. These data indicate that $\mathrm{O}_{2}{ }^{\bullet-}, \mathrm{H}_{2} \mathrm{O}_{2}$,

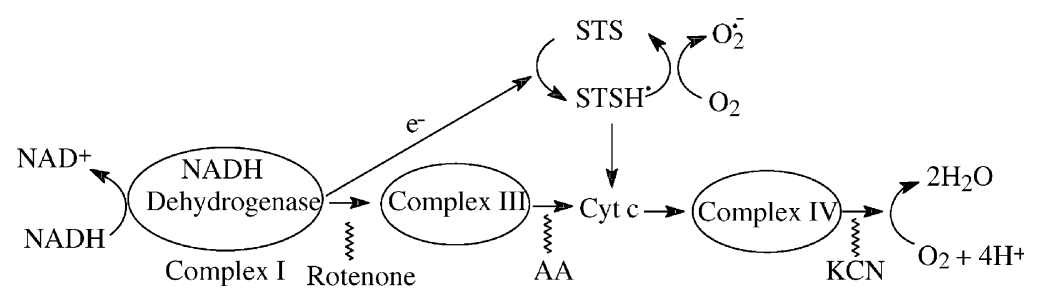

Fig. 9. A simplified schematic drawing of STS-mediating electron transport chain. This scheme shows that STS can accept one electron from mitochondrial complex I and then transfer the electron to oxygen through two ways. STS, sodium tanshinone IIA sulfonate; AA, antimycin A; Cyt c, cytochrome $c$. 
$\mathrm{OH}^{\bullet}$ all contribute to the chemiluminescence, but $\mathrm{O}_{2}{ }^{\bullet-}$ is the primary free radical in this process.

Based on the above, STS may mediate two different electron pathways in the presence of rotenone or antimycin A. Since the electron transfer rates of these two pathways were almost equal, the control step of STS-mediated pathway may accept electrons from complex I and form an intermediate. $\mathrm{K}_{3}\left[\mathrm{Fe}(\mathrm{CN})_{6}\right]$ is an artificial electron acceptor that can be reduced by NADH through mitochondrial complex I. We found that STS could inhibit this reduction in a dose-dependent manner. This observation further suggests that NADH dehydrogenase of complex I may be the site in which STS accepts electron while NADH is the electron donor.

In order to examine if the STS free radical was formed in above system, ESR spin trapping technique was used but no ESR signal was found, because the semiquinone free radical was not stable in physiological pH. If STS was first reduced by $\mathrm{NaBH}_{4}(1 \mathrm{mmol} / \mathrm{L})$ and then reoxidized by oxygen in alkaline condition, an absorption peak at $300 \mathrm{~nm}$ and a signal of free radical was found. Analysis of the ESR spectrum revealed that it was a semiquinone free radical of STS. Using pulse radiolysis technique, it was found that there was a transient peak at $300 \mathrm{~nm}$, and the reaction constant between hydroxyl radical and STS was about $1.8 \times 10^{9} \mathrm{dm}^{3} / \mathrm{mol} \mathrm{s}$. All these results suggest that STS can react with oxygen radicals and form a radical intermediate.

Our data suggest that oxygen free radicals were formed during STS mediated electron transport. Free radicals can initiate lipid peroxidation that may cause damage to tissue. Compared with the quinone form, the hydroquinone has a much higher antioxidative ability in scavenging ROS $[21,22]$. Since ROS were mainly formed during the reperfusion stage $[23,24]$, STS may be reduced to its hydroquinone form and scavenge ROS in ischemia-reperfusion tissue. Using ESR and pulse radiolysis techniques, we recognized a semiquinone free radical from a redox reaction of STS with ROS. In addition, by using the new electron pathway mediated by STS, tissue may maintain respiration under a low oxygen concentration.

\section{Acknowledgments}

We appreciate Dr. Qi Chen from the Salk Institute for Biological Studies for revising this manuscript. This work was supported by a grant from the National Natural Science Foundation of China.

\section{References}

[1] Shanghai Cooperative Group for the Study of Tanshinone IIA. Therapeutic effect of sodium tanshinone IIA sulfonate in patients with coronary heart diseases: a double blind study. J Trad Chin Med 1984;4:20-4.
[2] Chen W, Dong Y, Wang C, Ting G. Pharmacological studies of sodium tanshinone IIA sulfonate. Acta Pharmacol Sin 1979;14:277-83.

[3] Tao J, Wang QZ, Liu QY. Experimental study on prevent effect of tanshinone on injury of ischemia-reperfusion of heart. Acta Anaesthesiol Sin 1996;16:202-4.

[4] Zhao BL, Jiang W, Zhao Y, Hou JW, Xin WJ. Scavenging effects of Savia miltiorrhiza on free radical and its protection for myocardial mitochondrial membranes from ischemia-reperfusion injury. Biochem Mol Biol Int 1996;38:1171-82.

[5] Zhao Y, Jiang W, Hou JW, Zhao BL, Xin WJ. Effect of calcium overload and salviol (tanshinone) on the lipid free radical generated from lipid peroxidation of mitochondrial membrane. Acta Biochem Biophys Sin 1995;27:610-5.

[6] Xu CQ, Cang JS, He XM. Inhibition effect of tanshinone IIA on electron current of L-calcium in rat heart cell. Acta Pharmacol Toxicol Sin 1996;12:85-8.

[7] Li XH, Tong RCH. Relationship between inhibitory action of tanshinone on neutrophil function and its prophylactic effects on myocardial infarction. Acta Pharmacol Sin 1991;12:269-72.

[8] Ambrosio G, Zweier JL, Duilio C, Kuppusamy P, Santoro G, Elia PP, Tritto I, Cirillo P, Condorelli M, Chiariello M. Evidence that mitochondrial respiration is a source of potentially toxic oxygen free radicals in intact rabbit hearts subjected to ischemia and reflow. $\mathrm{J}$ Biol Chem 1993;268:18532-41.

[9] Kappus H, Sies H. Toxic drug effects associated with oxygen metabolism: redox cycling and lipid peroxidation. Experientia 1981;37: 1233-358.

[10] Davies KJ, Doroshow JH. Redox cycling of anthracyclines by cardiac mitochondria. I. Anthracycline radical formation by NADH dehydrogenase. J Biol Chem 1986;261:3060-7.

[11] Zhao BL, Xin WJ. Direct measurement of active oxygen free radical following reperfusion of ischemic rabbit myocardium with ESR technique. Chin Sci Bull 1990;35:56-9.

[12] Turrens JF, Beconi M, Barilla J, Chavez UB, McCord JM. Mitochondrial generation of oxygen radicals during reoxygenation of ischemic tissues. Free Radic Res Commun 1991;12/13:681-9.

[13] Zweier JL, Flaherty JT, Weisfeldt ML. Direct measurement of free radical generation following reperfusion of ischemic myocardium. Proc Natl Acad Sci USA 1987;84:1404-7.

[14] Tyler DD, Gonze J. The preparation of heart mitochondria from laboratory animals. Methods Enzymol 1967;10:75-7.

[15] Rasmussen UF, Rasmussen HN. NADH oxidase system (external) of muscle mitochondria and its role in the oxidation of cytoplasmic NADH. Biochem J 1985;229:631-41.

[16] Blair PV. The large-scale preparation and properties of heart mitochondria from slaughterhouse material. Methods Enzymol 1967;10:78-81.

[17] Smith AL. Preparation, properties and conditions for assay of mitochondria: slaughterhouse material, small-scale. Methods Enzymol 1967;10:81-6.

[18] Gornall AG, Bardawill CJ, David MM. Determination of serum proteins by means of the Biuret reaction. J Biol Chem 1949;177: 751-66.

[19] Ruzicka JK, Crane FL. Effect of antimycin on the potato mitochondrial cytochrome $b$ system. Biochim Biophys Acta 1970;223:71-85.

[20] Bemofsky C, Bandara BMR, Hinojosa Q. ESR studies of the reaction of hypochlorite with 5,5-dimethyl-1-pyrroline- $N$-oxide. Free Radic Biol Med 1990;8:231-9.

[21] Beyer REJ. The role of ascorbate in antioxidant protection of biomembrane: interaction with vitamin $\mathrm{E}$ and coenzyme Q. J Bioenerg Biomembr 1994;26:349-58.

[22] Ernster I, Dallner G. Biochemical, physiological and medical aspects of ubiquinone function. Biochim Biophys Acta 1995;1271:195-204.

[23] Flaherty JT, Weisfeldt ML. Reperfusion injury. Free Radic Biol Med 1988;5:409-19.

[24] Jamieson D. Oxygen toxicity and reactive oxygen metabolites in mammals. Free Radic Biol Med 1989;7:87-108. 